\title{
Evaluation for Peritoneal Injury at an Early Stage Using Dual Macromolecular Markers
}

\author{
Haruna Hirata, Shintaro Fumoto, Hirotaka Miyamoto, Mikiro Nakashima, Morio Nakayama, and \\ Koyo Nishida* \\ Graduate School of Biomedical Sciences, Nagasaki University; 1-7-1 Sakamoto, Nagasaki 852-8501, Japan. \\ Received December 24, 2015; accepted July 7, 2016
}

\begin{abstract}
Long-term peritoneal dialysis (PD) frequently produces morphological and functional changes of the peritoneum, making continuation of $\mathrm{PD}$ difficult. Therefore, it is necessary to evaluate peritoneal injury at an early stage and develop appropriate therapies. The aims of the present study were to evaluate peritoneal injury at an early stage and assess a drug for prevention of peritoneal injury using our previously developed novel evaluation method. Peritoneal injury was induced in model animals by intraperitoneal injection of methylglyoxal (MGO) for 1 to 5 consecutive days or chlorhexidine digluconate (CG) for 1 to 14 consecutive days. Tetramethylrhodamine-dextran (RD)-10 and fluorescein isothiocyanate-dextran (FD)-2000 were then injected into the peritoneal cavity and recovered after $120 \mathrm{~min}$ to evaluate peritoneal injury. The ratio of the concentration of RD-10 to FD-2000 (RD-10/FD-2000 ratio) significantly decreased in animals that had been treated with MGO or CG for $1 \mathrm{~d}$. Moreover, the RD-10/FD-2000 ratio significantly increased in CG- and thalidomide-treated animals. The RD-10/FD-2000 ratio can be used to evaluate peritoneal injury at an early stage and assess the drug efficacy of thalidomide for prevention of peritoneal injury. This study will contribute to the development of therapeutic treatments for peritoneal injury.
\end{abstract}

Key words early peritoneal injury; hyperpermeability; macromolecular marker; peritoneal dialysis

Peritoneal dialysis (PD) is a major treatment for end-stage renal disease and a useful therapy to compensate for some cases of renal function decline. In particular, PD can preserve residual renal function and contribute to improving the quality of life in patients undergoing PD. ${ }^{1-3)}$ In recent years, the "PD first," strategy, which involves starting with PD and then switching to hemodialysis after utilizing residual renal function, has been anticipated to provide a good prognosis for patients. However, long-term PD frequently produces morphological and functional changes of the peritoneum, making continuation of PD difficult. ${ }^{4-8)}$ Moreover, progression of peritoneal injury causes serious complications, such as encapsulating peritoneal sclerosis. ${ }^{9)}$ Therefore, it is necessary to evaluate peritoneal injury at an early stage and develop appropriate treatments for peritoneal injury.

Some recent studies have shown that several drugs have preventive effects for peritoneal injury. Rosiglitazone, a peroxisome proliferator-activated receptor- $\gamma$ (PPAR $\gamma$ ) agonist, reduced fibrosis and angiogenesis in two studies. ${ }^{10,11)}$ Reninangiotensin-aldosterone system-blocking drugs such as the renin inhibitor aliskiren, angiotensin-converting enzyme inhibitors, and angiotensin II receptor blockers improved peritoneal morphology and function in other studies. ${ }^{12-14)}$ Moreover, thalidomide prevented peritoneal fibrosis caused by chlorhexidine digluconate in rat and mouse experimental models. ${ }^{15,16)}$ However, preparation of animal models of peritoneal injury requires a long duration of time and great effort. Moreover, evaluation methods of drug efficacy for peritoneal injury such as the peritoneal equilibration test (PET), polymerase chain reaction, Western blot analysis, and immunohistochemical staining are problematic. The PET, which is the most widespread method for evaluation of peritoneal function, requires a long period of time to complete. Polymerase chain reaction, Western blot analysis, and immunohistochemical staining are complicated and high-cost.

We have developed an evaluation method for peritoneal injury using dual macromolecular markers: tetramethylrhodamine-dextran (RD-10) and fluorescein isothiocyanate-dextran (FD-2000). Our evaluation method utilizes the ratio of the concentrations of these two markers (the RD-10/FD-2000 ratio). ${ }^{17)} \mathrm{RD}-10$ in dialysate is suitable for evaluation of peritoneal injury, but the concentration of RD-10 is influenced by the transfer of water. FD-2000 compensates for the transfer of water. The RD-10/FD-2000 ratio can be used to easily evaluate peritoneal injury regardless of the drainage volume when the glucose concentration in the PD fluid (PDF) or the dosage volume has changed. When performing the PET, it is necessary to measure the concentrations of creatinine in the blood and PD effluent and glucose in the PD effluent. Because both creatinine and glucose are endogenous substances, the test data are influenced by the status of the patient. In contrast, our method uses exogenous substances; thus, the accuracy of our method may be superior to that of the PET. Moreover, the sensitivity of our method is high because of the measurement of fluorescence. Thus, we hypothesized that the RD-10/ FD-2000 ratio can be used to evaluate peritoneal injury at an early stage.

In this study, we evaluated rat and mouse models of shortduration peritoneal injury. Mice are easier to handle because their body weight is approximately one-tenth that of rats. We assessed whether the RD-10/FD-2000 ratio can be utilized as the evaluation method for peritoneal injury at an early stage. Moreover, we evaluated the use of thalidomide for prevention of peritoneal injury by the RD-10/FD-2000 ratio.

\section{MATERIALS AND METHODS}

Materials Methylglyoxal (MGO) was obtained from 
Nacalai Tesque, Inc. (Kyoto, Japan). Chlorhexidine digluconate $(C G)$ and FD were obtained from Sigma Chemical Co. (St. Louis, MO, U.S.A.). RD was obtained from Invitrogen (Carlsbad, CA, U.S.A.). Thalidomide and hematoxylin and eosin (H\&E) were obtained from Wako Pure Chemical Industries, Ltd. (Osaka, Japan). Embedding medium for frozen tissue specimens (Tissue-Tek O.C.T. compound) was obtained from Sakura Finetek Japan Co., Ltd. (Tokyo, Japan). The PDF (Dianeal- $N$ PD-4 1.5) was obtained from Baxter, Ltd. (Tokyo, Japan).

Animals Male Wistar rats (7 weeks old) and male ddY mice (5 weeks old) were housed in cages in an air-conditioned room and maintained on a standard laboratory diet (MF; Oriental Yeast Co., Ltd., Tokyo, Japan) and water ad libitum. All animal experiments in the present study conformed to the Guidelines for Animal Experimentation of Nagasaki University.

Preparation of Peritoneal Injury Animal Models Rats were prepared for peritoneal injury by the following slightly modified protocol. ${ }^{18)}$ Briefly, $20 \mathrm{~mL}$ of $20-\mathrm{mm}$ MGO dissolved in saline was injected intraperitoneally for 1 to 5 consecutive days. In a control experiment, saline was intraperitoneally injected in the same fashion. Mice were prepared for peritoneal injury by intraperitoneal injection of $2 \mathrm{~mL}$ of 20 - to $80-\mathrm{mm}$ MGO. Rats were prepared for CG-induced peritoneal injury by the following slightly modified protocol. ${ }^{15}$ ) Briefly, saline containing $0.1 \% \mathrm{CG}$ and $15 \%$ ethanol was injected intraperitoneally for 1 to 14 consecutive days at a volume of $10 \mathrm{~mL} /$ $\mathrm{kg}$ body weight. In a control experiment, saline containing $15 \%$ ethanol was intraperitoneally injected in the same fashion. Mice were prepared for CG-induced peritoneal injury by intraperitoneal injection of 0.01 to $0.1 \% \mathrm{CG}$ at a volume of $10 \mathrm{~mL} / \mathrm{kg}$ body weight.

Evaluation of Drug Efficacy Prevention of peritoneal injury was performed following a previously described protocol. ${ }^{15)}$ Thalidomide $(100 \mathrm{mg} / \mathrm{kg})$ was suspended in $0.5 \mathrm{~mL}$ olive oil and orally administered using a gastric tube once daily after the first administration of CG. In a control experiment, olive oil was orally administered in the same fashion.

Assessment of Peritoneal Injury with the RD-10/ FD-2000 Ratio Assessment of peritoneal injury with the RD-10/FD-2000 ratio was performed following a previously described protocol. ${ }^{17)}$ Rats or mice were anesthetized with sodium pentobarbital $(60 \mathrm{mg} / \mathrm{kg}$, intramuscular injection). The skin was cut and the abdominal wall exposed. FD-2000 (molecular weight (MW) 2000000, $0.05 \mathrm{mg}, 5 \mu \mathrm{g} / \mathrm{mL}$ ) and RD-10 (MW 10000, $0.1 \mathrm{mg}, 10 \mu \mathrm{g} / \mathrm{mL}$ ) dissolved in $10 \mathrm{~mL}$ of PDF (Dianeal- $N$ PD-4 1.5 containing 1.36\% glucose) were injected into the peritoneal cavity of the rats using a syringe with a 21-G $\times 1.5$-inch needle (Nipro Co., Ltd., Osaka, Japan). Alternatively, $1 \mathrm{~mL}$ of PDF was injected into the peritoneal cavity of the mice using a syringe with a $26-\mathrm{G} \times 0.5$-inch needle (Nipro Co., Ltd.). The needle was then removed, and the pinhole in the abdominal wall was closed with surgical adhesive (Aron Alpha A; Daiichi Sankyo Pharmaceutical Co., Ltd., Tokyo, Japan) to prevent leakage of the fluid. The entire residual solution in peritoneal cavity was recovered $120 \mathrm{~min}$ after the injection.

Quantification of Markers The obtained samples were centrifuged at $1600 \times \boldsymbol{g}$ at $4^{\circ} \mathrm{C}$ for $15 \mathrm{~min}$, and the supernatants were obtained. The quantification conditions were as follows.

For quantification of FD-2000, the sample $(20 \mu \mathrm{L})$ was diluted with $500 \mu \mathrm{L}$ of phosphate-buffered saline (PBS) and then measured using a spectrofluorophotometer (RF-1500; Shimadzu Corp., Kyoto, Japan) at excitation and emission wavelengths of 494 and $521 \mathrm{~nm}$, respectively. For quantification of RD-10, the sample $(40 \mu \mathrm{L})$ was diluted with $500 \mu \mathrm{L}$ of phosphate-buffered saline and then measured using the same spectrofluorophotometer at excitation and emission wavelengths of 555 and $580 \mathrm{~nm}$, respectively.

Histological Examination Histological analysis was performed by H\&E staining. After recovery of the residual solution in the peritoneal cavity, the abdominal walls were cut and fixed with $4 \%$ paraformaldehyde in phosphate buffer. They were then embedded in O.C.T. compound frozen at $-80^{\circ} \mathrm{C}$, and sliced into $10-\mu \mathrm{m}$ sections using a microtome (Retoratome REM-710; Yamato Kohki Industrial Co., Ltd., Saitama, Japan) and stained by H\&E. Stained samples were observed using a microscope with a $10 \times$ objective lens.

Statistical Analyses Statistical comparisons between two groups were performed by an unpaired Student's $t$-test (Figs. $1,3,5 \mathrm{a}, 5 \mathrm{c}, 7,8)$, and multiple comparisons were performed by two-way ANOVA followed by Dunnett's test (Figs. 5b, d). A $p$ value of $<0.05$ was considered to indicate statistical significance.
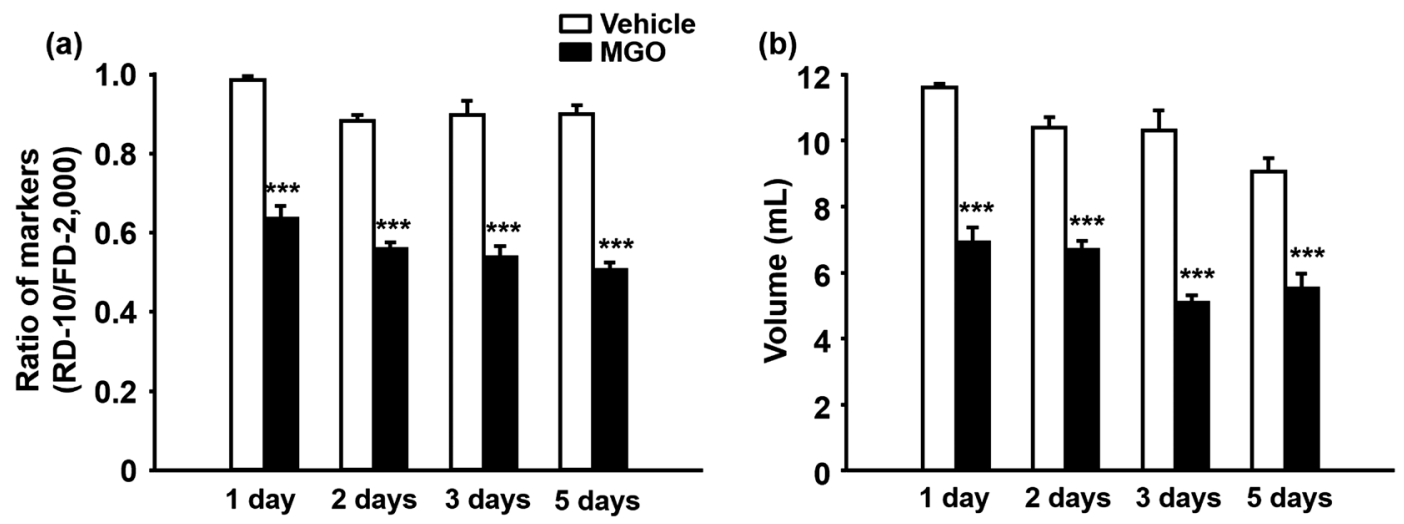

Fig. 1. RD-10/FD-2000 Ratio and Recovery Volume of Peritoneal Fluid in MGO-Treated Rats

(a) Evaluation of peritoneal injury using the RD-10/FD-2000 ratio, and (b) the residual solution in the peritoneal cavity 120 min after intraperitoneal injection in rats treated with saline or MGO $(20 \mathrm{~mm})$ for 1 to 5 consecutive days. The 5 -d data are quoted from ref. 17. Each bar represents the mean+S.E. for at least four experiments. $* * * p<0.001$ compared with saline-treated rats. 


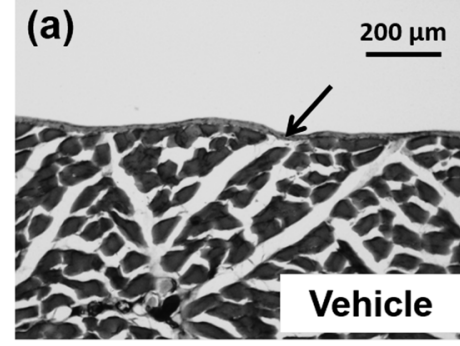

(d)

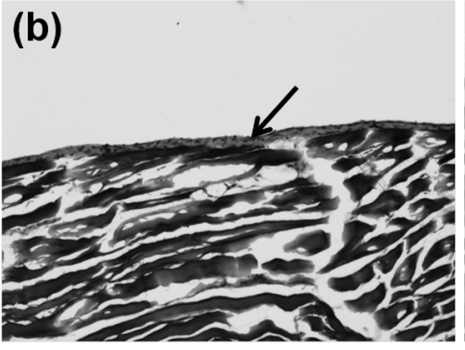

(e)

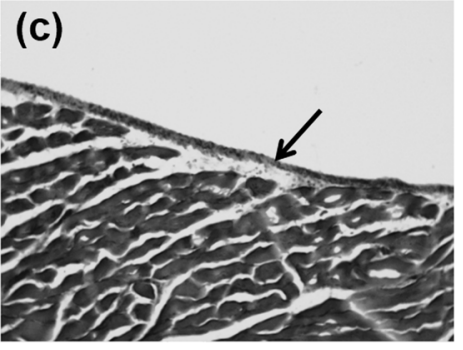

(f)
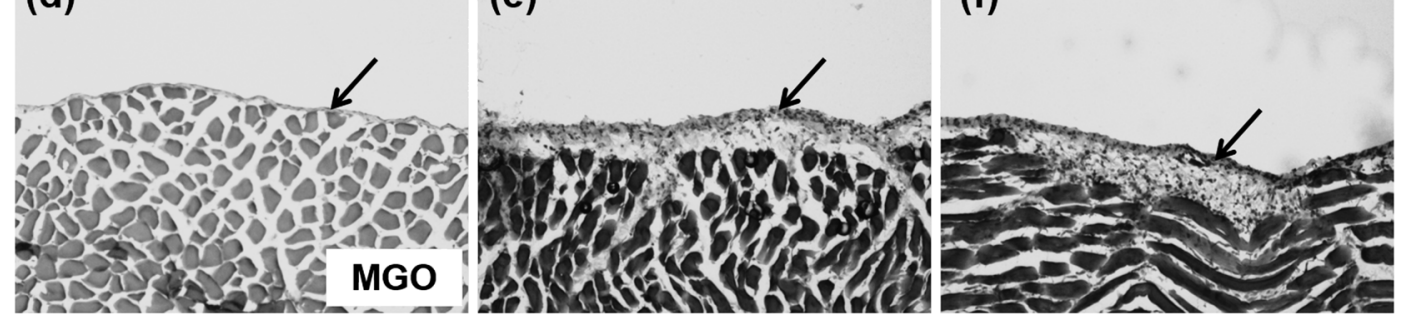

Fig. 2. Morphological Changes of the Peritoneum in MGO-Treated Rats

Observation of the peritoneum of rats treated with saline for (a) 1, (b) 2, and (c) 3 consecutive days. Peritoneum of rats treated with MGO (20 mm) for (d) 1, (e) 2, and (f) 3 consecutive days. Arrows indicate the peritoneum.
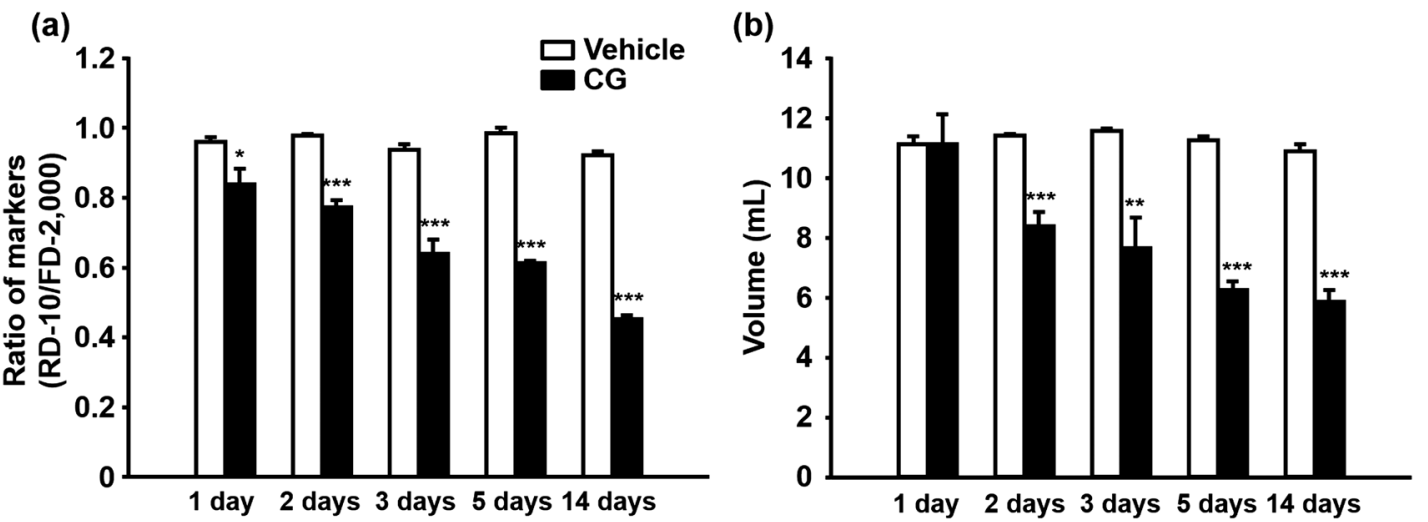

Fig. 3. RD-10/FD-2000 Ratio and Recovery Volume of Peritoneal Fluid in CG-Treated Rats

(a) Evaluation of peritoneal injury using the RD-10/FD-2000 ratio, and (b) the residual solution in the peritoneal cavity 120 min after intraperitoneal injection in rats treated with $15 \%$ ethanol or CG $(0.1 \%)$ for 1 to 14 consecutive days. Each bar represents the mean + S.E. for at least four experiments. $* p<0.05, * * p<0.01$, $* * * p<0.001$ compared with $15 \%$ ethanol-treated rats.

\section{RESULTS}

Evaluation Method for Peritoneal Injury at an Early Stage To evaluate peritoneal injury at an early stage using the RD-10/FD-2000 ratio, MGO (which is generally used for 5 consecutive days to induce peritoneal injury) or CG (which is generally used for 14 consecutive days to cause peritoneal injury) was administered to the rats for a short period. The RD-10/FD-2000 ratio significantly decreased in rats treated with MGO for 1 to 5 consecutive days compared with those in saline (vehicle)-treated rats (Fig. 1a). The recovery volume of peritoneal fluid $120 \mathrm{~min}$ after injection was also significantly decreased in MGO-treated rats (Fig. 1b). Figure 2 shows the peritoneum of vehicle-treated and MGO-treated rats by H\&E staining. The peritoneum of rats treated with MGO for $1 \mathrm{~d}$ did not exhibit an altered morphology, but that in rats treated with MGO for 2 and 3 consecutive days was thick (Fig. 2). Furthermore, the RD-10/FD-2000 ratio significantly decreased in rats treated with $\mathrm{CG}$ for 1 to 14 consecutive days compared with vehicle-treated rats (Fig. 3a). However, the recovery volume of peritoneal fluid did not decrease in the rats treated with $\mathrm{CG}$ for $1 \mathrm{~d}$ (Fig. 3b). The peritoneum of rats treated with $\mathrm{CG}$ for 2 to $14 \mathrm{~d}$ exhibited an altered morphology, but those of rats treated with $\mathrm{CG}$ for $1 \mathrm{~d}$ and vehicle-treated rats did not change (Fig. 4).

Mice were treated with MGO or CG to develop an easy evaluation method for peritoneal injury. The RD-10/FD-2000 ratio significantly decreased in mice treated with MGO for 1 to 5 consecutive days compared with that in vehicle-treated mice (Fig. 5a). In addition, a high concentration of MGO (25-80 mM) was administered to mice for $1 \mathrm{~d}$ because the difference in the RD-10/FD-2000 ratio in mice treated with 20-mm MGO for $1 \mathrm{~d}$ was small. The RD-10/FD-2000 ratio significantly decreased in MGO-treated mice and was minimal in those treated with 40-mm MGO (Fig. 5b). Figure 5c shows the RD-10/FD-2000 ratios of the mice treated with CG for 1 to 14 consecutive days. These ratios significantly decreased in the CG-treated mice (Fig. 5c). Moreover, a low concentration of CG $(0.01-0.05 \%)$ was administered to the mice for $1 \mathrm{~d}$. The RD-10/FD-2000 ratio significantly decreased in mice treated with $>0.03 \%$ CG (Fig. 5d).

Evaluation of Drug Efficacy for Prevention of Peritoneal 


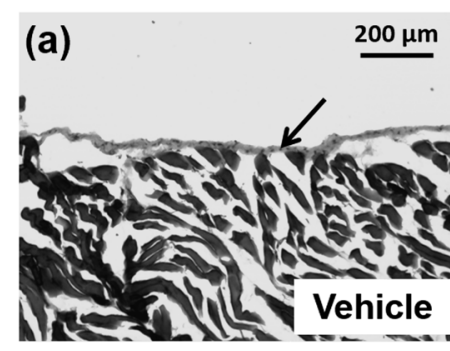

(d)

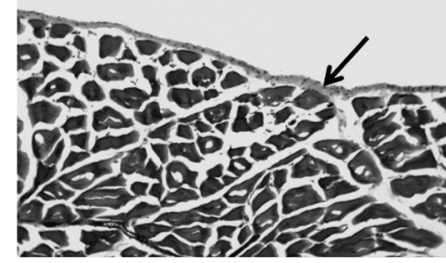

(f)

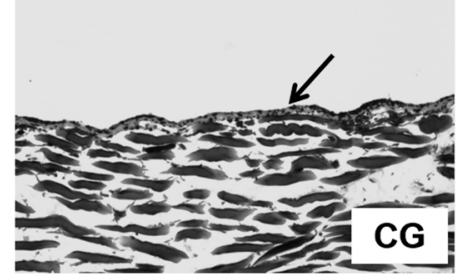

(i)

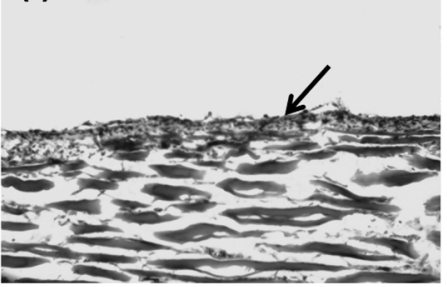

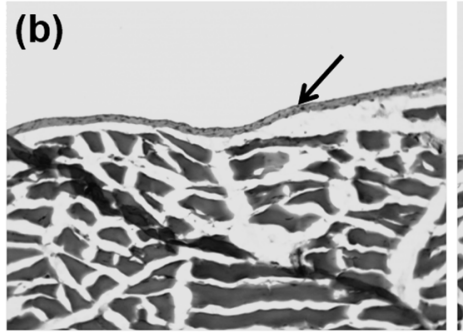

(e)

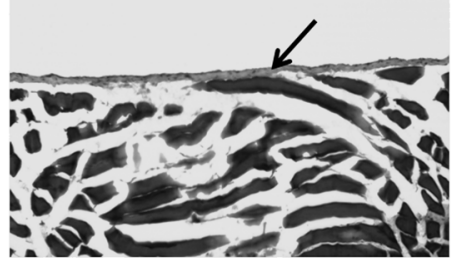

(g)

(h)
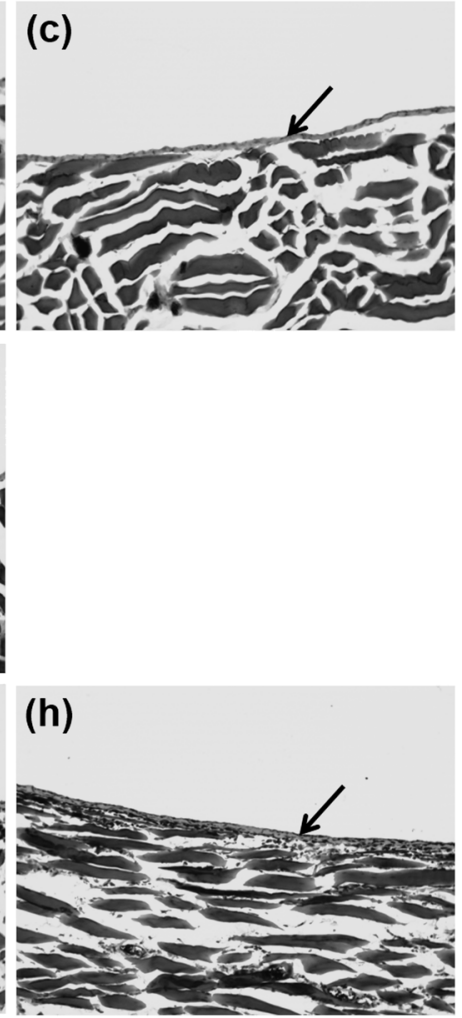

(j) (n) 

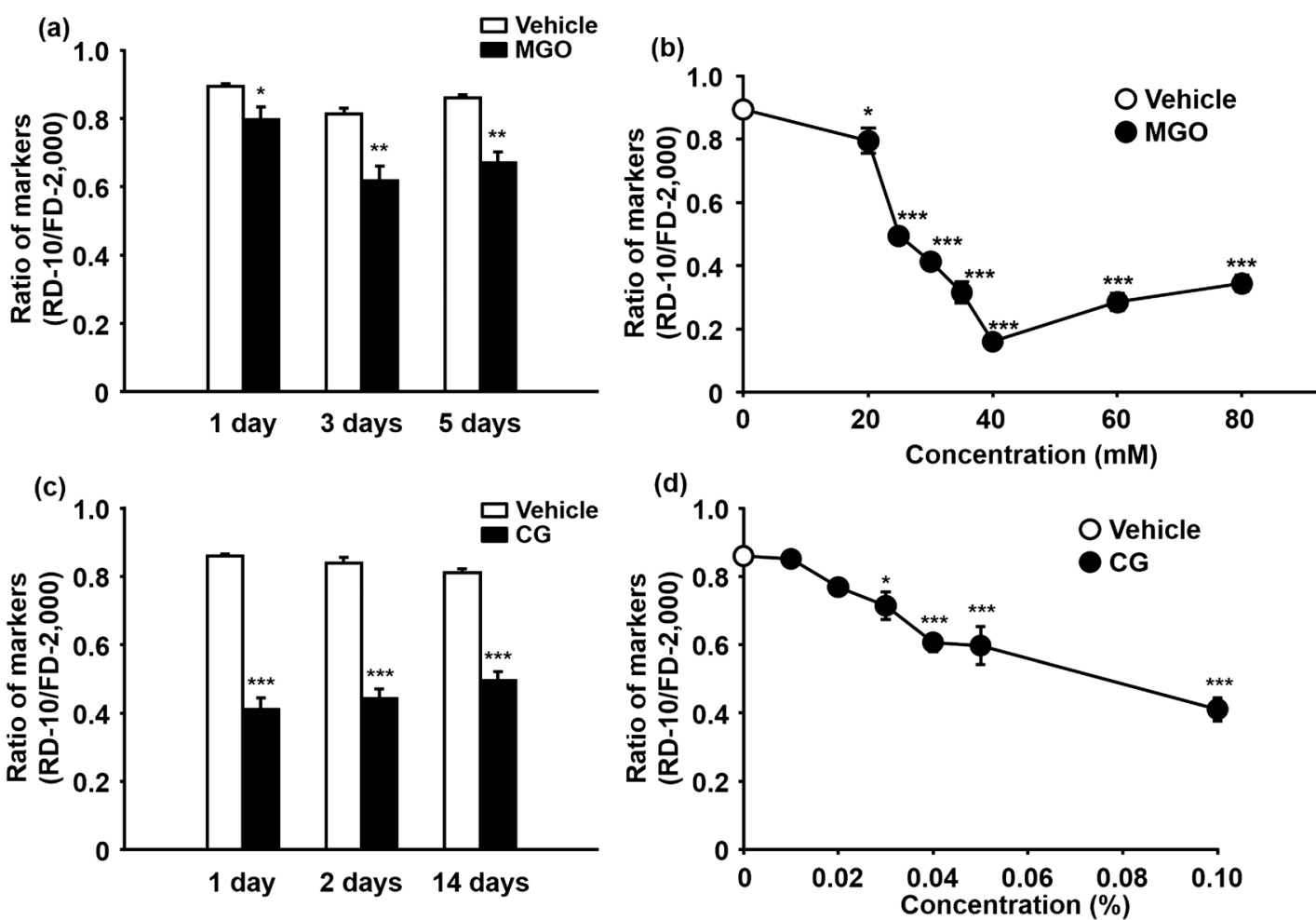

Fig. 5. RD-10/FD-2000 Ratio in MGO- or CG-Treated Mice

(a) Evaluation of peritoneal injury using the RD-10/FD-2000 ratio in mice treated with MGO (20 mM) for 1 to 5 consecutive days, (b) a high concentration of MGO $(25-80 \mathrm{~mm})$ for $1 \mathrm{~d}$, (c) CG $(0.1 \%)$ for 1 to 14 consecutive days, and (d) a low concentration of CG $(0.01-0.05 \%)$ for $1 \mathrm{~d}$. Each bar represents the mean + S.E. or \pm S.E. for at least four experiments. ${ }^{*} p<0.05, * * p<0.01, * * * p<0.001$ compared with vehicle-treated mice.

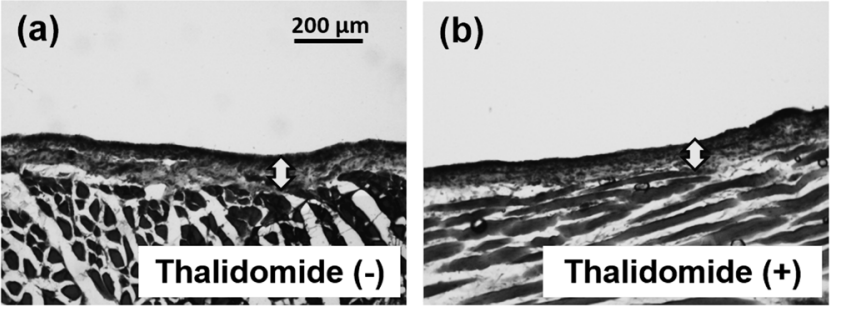

Fig. 6. Peritoneum of Rats Administered Thalidomide

(a) Peritoneum of rats treated with CG $(0.1 \%)$ for 14 consecutive days. (b) Peritoneum of rats treated with CG $(0.1 \%)$ and thalidomide for 14 consecutive days. Arrows indicate the peritoneum.

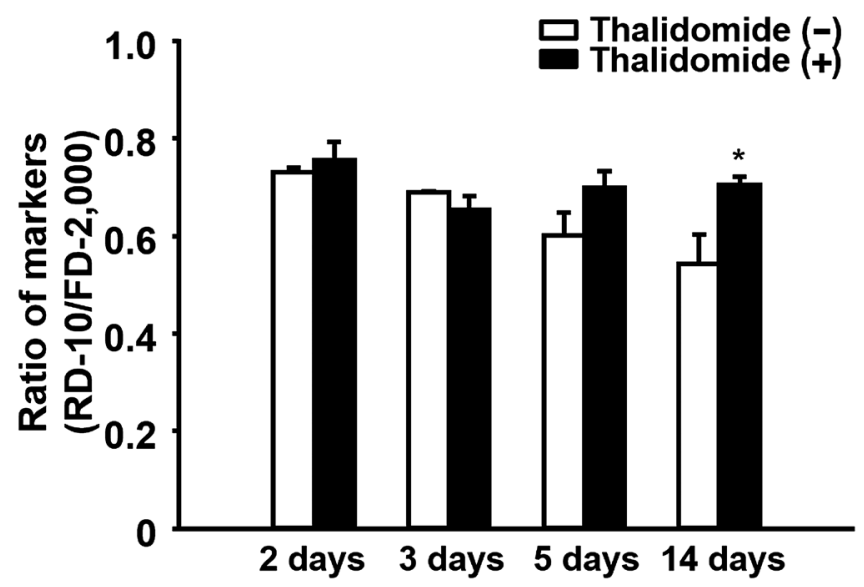

Fig. 7. RD-10/FD-2000 Ratio of Rats Administered Thalidomide

Evaluation of peritoneal injury using the RD-10/FD-2000 ratio in rats treated with CG $(0.1 \%)$ and thalidomide for 2 to $14 \mathrm{~d}$. Each bar represents the mean+S.E. for at least four experiments. ${ }^{*} p<0.05$ compared with vehicle-administered rats.

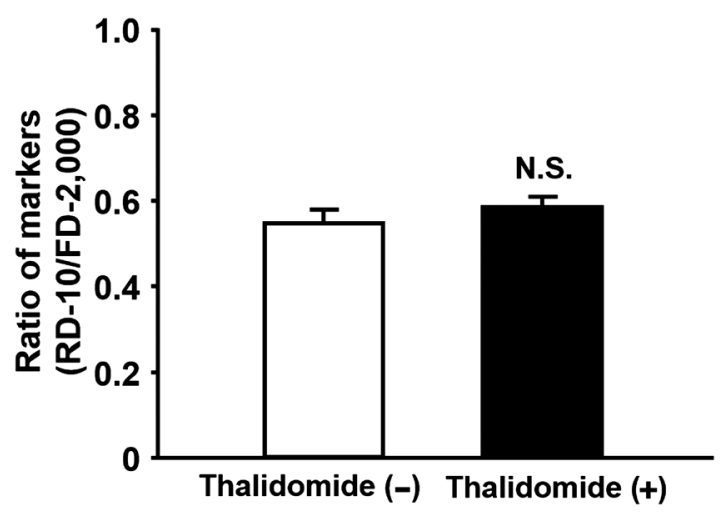

Fig. 8. RD-10/FD-2000 Ratio in Mice Administered Thalidomide

Evaluation of peritoneal injury using the RD-10/FD-2000 ratio in mice treated with CG $(0.1 \%)$ and thalidomide for $1 \mathrm{~d}$. Each bar represents the mean + S.E. for at least eight experiments. N.S., not significant.

may be induced after production of these cytokines, and the RD-10/FD-2000 ratio might therefore be useful for evaluation of peritoneal injury at an early stage.

We prepared a mouse model of peritoneal injury to develop an easier evaluation method than the conventional methods currently in use. Because the body weight of mice is one-tenth that of rats, it is easy to handle mice and decrease the amount of drugs during evaluation of drug efficacy. The RD-10/ FD-2000 ratio in mice treated with MGO or CG for $1 \mathrm{~d}$ was significantly decreased (Figs. 5a, c). This result suggests that the RD-10/FD-2000 ratio can be used to evaluate peritoneal injury at an early stage in mice. Moreover, when several concentrations of MGO or CG were injected for $1 \mathrm{~d}$, the RD-10/ FD-2000 ratio gradually decreased as the concentration of 
MGO or CG increased (Figs. 5b, d). This suggests that we can induce various degrees of peritoneal injury in mice within a short duration of time.

The use of biocompatible PDFs reportedly has various benefits such as reduction of the epithelial-to-mesenchymal transition in peritoneal mesothelial cells and prevention of peritoneal fibrosis; however, it does not readily prevent peritoneal injury completely. ${ }^{21,22)}$ Thus, it is necessary to develop therapeutic treatments for peritoneal injury. Some studies have suggested that several drugs have therapeutic effects for peritoneal injury. ${ }^{10-16)}$ We evaluated the effect of thalidomide for prevention of peritoneal injury by the RD-10/FD-2000 ratio. Thalidomide is a useful therapeutic drug for peritoneal injury in animal experiments because it prevents peritoneal fibrosis. Moreover, thalidomide has been approved as a therapeutic drug for multiple myeloma and erythema nodosum leprosum, and it is utilized in clinical practice. Although we evaluated peritoneal injury in rats treated by thalidomide following a previous report ${ }^{15)}$ the thickness of the peritoneum was indistinguishable from that in rats not treated with thalidomide (Fig. 6). However, the RD-10/FD-2000 ratio was significantly increased in rats treated with thalidomide for $14 \mathrm{~d}$ (Fig. 7), indicating that the RD-10/FD-2000 ratio can be used to evaluate the drug efficacy of thalidomide, which cannot be distinguished at the morphological level. Here, conventional preparation of animal models of peritoneal injury using MGO (which is a glucose degradation product), ${ }^{23,24)} \mathrm{CG}^{25)}$ or $\mathrm{PDF}^{26,27)}$ requires a long duration of time and great effort (MGO is administered for 5 consecutive days, CG is administered for more than 14 consecutive days, and PDF is administered for more than 28 consecutive days). It is thought to be difficult to quickly evaluate drug efficacy for peritoneal injury by conventional evaluation methods. Then, we evaluated the efficacy of short-term administration of thalidomide by the RD-10/FD-2000 ratio. An effect of $1 \mathrm{~d}$ of thalidomide administration was not observed in mice (Fig. 8) because CG induced strong peritoneal injury immediately (Fig. 5c). This result suggests that the effects of thalidomide are gradual. Taking all results into consideration, the RD-10/FD-2000 ratio may be used to explore effective drugs for peritoneal injury.

In conclusion, our findings indicate that the RD-10/ FD-2000 ratio can be used to evaluate peritoneal injury at an early stage. Moreover, the RD-10/FD-2000 ratio can be used to evaluate the efficacy of thalidomide for prevention of peritoneal injury. Therefore, we might be able to prepare model animals for one day and evaluate drug efficacy for peritoneal injury. This study will contribute to the development of effective treatments for peritoneal injury.

Acknowledgments This work was supported in part by a Grant-in-Aid for Scientific Research from the Ministry of Education, Culture, Sports, Science and Technology of Japan and by the Joint Research Promotion Project of Nagasaki University Graduate School of Biomedical Sciences in 2013.

Conflict of Interest The authors declare no conflict of interest.

\section{REFERENCES}

1) Lameire NH. The impact of residual renal function on the adequacy of peritoneal dialysis. Nephron, 77, 13-28 (1997).

2) Noshad H, Sadreddini S, Nezami N, Salekzamani Y, Ardalan MR Comparison of outcome and quality of life: Haemodialysis versus peritoneal dialysis patients. Singapore Med. J., 50, 185-192 (2009).

3) Chaudhary K, Sangha H, Khanna R. Peritoneal dialysis first: Rationale. Clin. J. Am. Soc. Nephrol., 6, 447-456 (2011).

4) Fusshoeller A. Histomorphological and functional changes of the peritoneal membrane during long-term peritoneal dialysis. Pediatr. Nephrol., 23, 19-25 (2008).

5) Shimaoka T, Hamada C, Kaneko K, Io H, Sekiguchi Y, Aruga S, Inuma J, Inami Y, Hotta Y, Horikoshi S, Kumasaka T, Tomino Y. Quantitative evaluation and assessment of peritoneal morphologic changes in peritoneal dialysis patients. Nephrol. Dial. Transplant., 25, 3379-3385 (2010).

6) Davies SJ. Longitudinal relationship between solute transport and ultrafiltration capacity in peritoneal dialysis patients. Kidney Int., 66, 2437-2445 (2004).

7) $\mathrm{Oh} \mathrm{KH}$, Jung JY, Yoon MO, Song A, Lee H, Ro H, Hwang YH, Kim DK, Margetts P, Ahn C. Intra-peritoneal interleukin-6 system is a potent determinant of the baseline peritoneal solute transport in incident peritoneal dialysis patients. Nephrol. Dial. Transplant., 25, 1639-1646 (2010)

8) Boulanger E, Wautier MP, Gane P, Mariette C, Devuyst O, Wautier JL. The triggering of human peritoneal mesothelial cell apoptosis and oncosis by glucose and glycoxydation products. Nephrol. Dial. Transplant., 19, 2208-2216 (2004).

9) Honda K, Oda H. Pathology of encapsulating peritoneal sclerosis. Perit. Dial. Int., 25 (Suppl. 4), S19-S29 (2005).

10) Bozkurt D, Taskin H, Sezak M, Biçak S, Sen S, Ok E, Duman S. Rosiglitazone, a peroxisome proliferator-activated receptor agonist, improves peritoneal alterations resulting from an encapsulated peritoneal sclerosis model. Adv. Perit. Dial., 24, 32-38 (2008).

11) Sandoval P, Loureiro J, González-Mateo G, Pérez-Lozano ML, Maldonado-Rodríguez A, Sánchez-Tomero JA, Mendoza L, Santamaría B, Ortiz A, Ruíz-Ortega M, Selgas R, Martín P, Sánchez-Madrid F, Aguilera A, López-Cabrera M. PPAR- $\gamma$ agonist rosiglitazone protects peritoneal membrane from dialysis fluid-induced damage. $L a b$. Invest., 90, 1517-1532 (2010).

12) Pérez-Martínez J, Pérez-Martínez FC, Carrión B, Masiá J, Ortega A, Simarro E, Nam-Cha SH, Ceña V. Aliskiren prevents the toxic effects of peritoneal dialysis fluids during chronic dialysis in rats. PLoS ONE, 7, e36268 (2012).

13) Bozkurt D, Cetin P, Sipahi S, Hur E, Nar H, Ertilav M, Sezak M, Duman S. The effects of renin-angiotensin system inhibition on regression of encapsulating peritoneal sclerosis. Perit. Dial. Int., 28 (Suppl. 5), S38-S42 (2008).

14) Lee CJ, Subeq YM, Lee RP, Ke CY, Lin NT, Hsu BG. Beneficial effects of enalapril on chlorhexidine digluconate-induced liver peritoneal fibrosis in rats. Chin. J. Physiol., 54, 225-234 (2011).

15) Mondello S, Mazzon E, Di Paola R, Crisafulli C, Mondello P, Buemi M, Aloisi C, Cuzzocrea S. Thalidomide suppresses sclerosing encapsulating peritonitis in a rat experimental model. Shock, 32, 332-339 (2009).

16) Arai H, Furusu A, Nishino T, Obata Y, Nakazawa $Y$, Nakazawa M, Hirose M, Abe K, Koji T, Kohno S. Thalidomide prevents the progression of peritoneal fibrosis in mice. Acta Histochem. Cytochem., 44, 51-60 (2011).

17) Hirata H, Miyamoto H, Shimokawa K, Nakashima M, Nakayama M, Fumoto S, Nishida K. Novel diagnostic method of peritoneal injury using dual macromolecular markers. Biol. Pharm. Bull., 37, 262-267 (2014).

18) Nakayama M, Sakai A, Numata M, Hosoya T. Hyper-vascular change and formation of advanced glycation endproducts in the peritoneum caused by methylglyoxal and the effect of an antioxidant, sodium sulfite. Am. J. Nephrol., 23, 390-394 (2003). 19) Suga H, Teraoka S, Ota K, Komemushi S, Furutani S, Yamauchi S, 
Margolin S. Preventive effect of pirfenidone against experimental sclerosing peritonitis in rats. Exp. Toxicol. Pathol., 47, 287-291 (1995).

20) Mizuiri S, Ohashi $Y$, Hemmi H, Arita M, Yamada K, Aoki T, Miyagi M, Sakai K, Aikawa A. Effects of new peritoneal dialysis solutions, pyridoxamine and AT1 receptor blocker, on TGF-beta1 and VEGF expression in rat peritoneal mesothelial cells. Am. J. Nephrol., 30, 295-302 (2009).

21) Fernández-Perpén A, Pérez-Lozano ML, Bajo MA, Albar-Vizcaino P, Sandoval Correa P, del Peso G, Castro MJ, Aguilera A, Ossorio M, Peter ME, Passlick-Deetjen J, Aroeira LS, Selgas R, LópezCabrera M, Sánchez-Tomero JA. Influence of bicarbonate/low-GDP peritoneal dialysis fluid (BicaVera) on in vitro and ex vivo epithelial-to-mesenchymal transition of mesothelial cells. Perit. Dial. Int., 32, 292-304 (2012).

22) Kawanishi K, Honda K, Tsukada M, Oda H, Nitta K. Neutral solution low in glucose degradation products is associated with less peritoneal fibrosis and vascular sclerosis in patients receiving peri- toneal dialysis. Perit. Dial. Int., 33, 242-251 (2013).

23) Hirahara I, Kusano E, Yanagiba S, Miyata Y, Ando Y, Muto S, Asano Y. Peritoneal injury by methylglyoxal in peritoneal dialysis. Perit. Dial. Int., 26, 380-392 (2006).

24) Hirahara I, Ishibashi Y, Kaname S, Kusano E, Fujita T. Methylglyoxal induces peritoneal thickening by mesenchymal-like mesothelial cells in rats. Nephrol. Dial. Transplant., 24, 437-447 (2009).

25) Hoff CM. Experimental animal models of encapsulating peritoneal sclerosis. Perit. Dial. Int., 25 (Suppl. 4), S57-S66 (2005).

26) Zareie M, Keuning ED, ter Wee PM, Beelen RH, van den Born J. Peritoneal dialysis fluid-induced changes of the peritoneal membrane are reversible after peritoneal rest in rats. Nephrol. Dial. Transplant., 20, 189-193 (2005).

27) Fabbrini P, Schilte MN, Zareie M, ter Wee PM, Keuning ED, Beelen RH, van den Born J. Celecoxib treatment reduces peritoneal fibrosis and angiogenesis and prevents ultrafiltration failure in experimental peritoneal dialysis. Nephrol. Dial. Transplant., 24, 3669-3676 (2009). 